\title{
ChIP-chip versus ChIP-seq: Lessons for experimental design and data analysis
}

\author{
Joshua WK Ho ${ }^{1,2}$, Eric Bishop ${ }^{2,3}$, Peter V Karchenko ${ }^{1,2,5}$, Nicolas Nègre ${ }^{4}$, Kevin P White ${ }^{4}$, Peter J Park ${ }^{1,2,5^{*}}$
}

\begin{abstract}
Background: Chromatin immunoprecipitation (ChIP) followed by microarray hybridization (ChIP-chip) or highthroughput sequencing (ChIP-seq) allows genome-wide discovery of protein-DNA interactions such as transcription factor bindings and histone modifications. Previous reports only compared a small number of profiles, and little has been done to compare histone modification profiles generated by the two technologies or to assess the impact of input DNA libraries in ChIP-seq analysis. Here, we performed a systematic analysis of a modENCODE dataset consisting of 31 pairs of ChIP-chip/ChIP-seq profiles of the coactivator CBP, RNA polymerase II (RNA PollI), and six histone modifications across four developmental stages of Drosophila melanogaster.

Results: Both technologies produce highly reproducible profiles within each platform, ChIP-seq generally produces profiles with a better signal-to-noise ratio, and allows detection of more peaks and narrower peaks. The set of peaks identified by the two technologies can be significantly different, but the extent to which they differ varies depending on the factor and the analysis algorithm. Importantly, we found that there is a significant variation among multiple sequencing profiles of input DNA libraries and that this variation most likely arises from both differences in experimental condition and sequencing depth. We further show that using an inappropriate input DNA profile can impact the average signal profiles around genomic features and peak calling results, highlighting the importance of having high quality input DNA data for normalization in ChIP-seq analysis.

Conclusions: Our findings highlight the biases present in each of the platforms, show the variability that can arise from both technology and analysis methods, and emphasize the importance of obtaining high quality and deeply sequenced input DNA libraries for ChIP-seq analysis.
\end{abstract}

\section{Background}

Chromatin immunoprecipitation (ChIP) followed by genomic tiling microarray hybridization (ChIP-chip) or massively parallel sequencing (ChIP-seq) are two of the most widely used approaches for genome-wide identification and characterization of in vivo protein-DNA interactions. They can be used to analyze many important DNA-interacting proteins including RNA polymerases, transcription factors, transcriptional co-factors, and histone proteins [1]. Indeed these genome-wide ChIP analysis approaches have led to many important discoveries related to transcriptional regulation [2-4], epigenetic regulation through histone modification [5],

\footnotetext{
* Correspondence: peter_park@harvard.edu

'Department of Medicine, Brigham and Women's Hospital, and Harvard Medical School, Boston, MA, USA

Full list of author information is available at the end of the article
}

nucleosome organization [6,7], and interindividual variation in protein-DNA interactions $[8,9]$.

ChIP-chip first appeared in the literature about 10 years ago and was one of the earliest approaches to performing genome-wide mapping of protein-DNA interactions in organisms with small genomes, such as yeast $[2,10]$. Currently, various tiling microarray platforms of common model organisms are well supported by commercial vendors, and many bioinformatics tools have been developed for ChIP-chip analysis [11-14]. Fueled by rapid development of the second generation high-throughput sequencing technologies in the past few years, ChIP-seq has emerged as an attractive alternative to ChIP-chip [1]. For instance, ChIP-seq generally produces profiles with higher spatial resolution, dynamic range, and genomic coverage, allowing it to have higher sensitivity and specificity over ChIP-chip in terms of protein binding site identification. Further, ChIP-seq can
C Biomed Central

() 2011 Ho et al; licensee BioMed Central Ltd. This is an Open Access article distributed under the terms of the Creative Commons Attribution License (http://creativecommons.org/licenses/by/2.0), which permits unrestricted use, distribution, and reproduction in any medium, provided the original work is properly cited. 
be used to analyze virtually any species with a sequenced genome since it is not constrained by the availability of an organism-specific microarray. Many current ChIP-seq protocols can work with a smaller amount of initial material compared to ChIP-chip $[15,16]$. Moreover, ChIP-seq is already a more costeffective way of analyzing mammalian genomes, and the cost effectiveness will likely become more apparent as the cost of high-throughput sequencing technology continues to drop. These factors have led to the rapid adoption of ChIP-seq technology.

However, despite the widespread use of both ChIPchip and ChIP-seq, only a few small-scale studies have attempted to quantitatively compare these technologies using real data. Euskirchen et al. [17] compared the STAT1 binding sites identified by ChIP-chip and ChIPPET (paired-end ditag sequencing by Sanger sequencing technology) and found that there was a good overall agreement between the two technologies, particularly at identifying highly ranked enrichment regions. They nonetheless noted specific discrepancies in regions associated with repetitive elements, which can be attributed to lack of microarray probe coverage or misalignment of ChIP-PET reads. More recently, a number of studies compared genome-wide transcription factor binding datasets generated from ChIP-chip with those generated from ChIP-seq [18-22] (see Additional file 1: Table S1). The general conclusions from these studies were that binding profiles generated from ChIP-chip and ChIP-seq were largely correlated at the genome-wide level, and that ChIP-seq had superior sensitivity and specificity over ChIP-chip in terms of binding site identification as determined by motif enrichment or quantitative PCR validation. It was also found that the strongest peaks were more likely to be detected by both technologies. However, only a few pairs of ChIP-chip/ChIP-seq profiles were analyzed in these studies, and their focus was mainly on the ability to identify narrow enrichment regions using specific peak calling algorithms. As shown previously [23] and in this study, peak identification can be strongly dependent on the analysis algorithm, so other more general comparison metrics should be used.

In addition, little is known about the technologyspecific variation for analyzing histone modification data. ChIP-based histone modification data is commonly used to reconstruct average signal profiles, or "epigenetic signatures," of key genomic regions such as the transcription start and end sites, but the impact of using ChIP-chip versus ChIP-seq data for constructing epigenetic signatures is largely unknown. Furthermore, it is also important to understand technology-specific biases associated with high-throughput sequencing. Recent studies indicated that the distribution of cross-linked and sonicated DNA fragments (input DNA) was affected by chromatin structure, copy number variation, occurrence of genomic repeats, mappability, genomic location, gene expression activity, and genomic GC content variation [24-26]. Since input DNA is commonly used as a background control for a ChIP-seq experiment, it is important to assess how such variation affects the analysis of ChIP-seq data.

Therefore a thorough understanding of the technological variation between ChIP-chip and ChIP-seq is important in experimental design and data analysis. In this study, we compiled and analyzed 31 pairs of ChIPchip/ChIP-seq profiles of technical replicates across eight immunoprecipitation (IP) factors (CBP, RNA PolII, and six histone modifications) at four developmental stages of the common fruit fly Drosophila melanogaster (Table 1) as part of the model organism Encyclopedia of DNA Elements (modENCODE) project [27]. In addition, our compiled dataset comprises another 62 ChIP-chip profiles (biological replicates) in the same set of biological conditions (i.e., three ChIP-chip biological replicates at each developmental stage/IP combination), nine sequencing profiles of input DNA, and four pairs of ChIP-seq/ChIP-seq replicates (Table 2). Agilent's tiling microarray (Agilent custom 3X244K Dmel Whole Genome Tiling Microarray) and Illumina's Genome Analyzer II platforms were used to generate the ChIP-chip and ChIP-seq data, respectively. All data used in this study were generated as part of the modENCODE project, and are accessible from NCBI GEO (accession numbers: GSE15292, GSE16013, and GSE20000). The goal of this study was to quantify reproducibility within

Table 1 Summary of the ChIP-chip and ChIP-seq profiles analyzed in this study

\begin{tabular}{rcccccccc}
\hline \multicolumn{1}{c}{ ChIP-chip and ChIP-seq replicates } \\
\hline IP factor & E-0-4 h & E-12-16 h & E-16-20 h & E-20-24 h \\
& chip & seq & chip & seq & chip & seq & chip & seq \\
\hline CBP & 3 & 1 & - & - & 3 & 1 & 3 & 1 \\
H3K27AC & 3 & 1 & 3 & 1 & 3 & 1 & 3 & 1 \\
H3K27Me3 & 3 & 1 & 3 & 1 & 3 & 1 & 3 & 1 \\
H3K4Me1 & 3 & 1 & 3 & 1 & 3 & 1 & 3 & 1 \\
H3K4Me3 & 3 & 1 & 3 & 1 & 3 & 1 & 3 & 1 \\
H3K9AC & 3 & 1 & 3 & 1 & 3 & 1 & 3 & 1 \\
H3K9Me3 & 3 & 1 & 3 & 1 & 3 & 1 & 3 & 1 \\
Poll & 3 & 1 & 3 & 1 & 3 & 1 & 3 & 1 \\
INPUT & $*$ & 1 & $*$ & 1 & $*$ & 1 & $*$ & 1 \\
\hline
\end{tabular}

For each combination of developmental stage and IP factor, three biological samples were profiled by ChIP-chip, and one of these samples was independently profiled by ChIP-seq. Therefore we have three biological ChIPchip/ChIP-chip replicates, and one technical ChIP-chip/ChIP-seq replicate pair for every biological condition. *Although no independent microarray profiles of input DNA were available, we extracted input DNA profiles from the input channel (of the two-channel microarray) for each ChIP-chip profile. We therefore have $31 \times 3=93$ INPUT-chip profiles in this collection, where all INPUT-chip profiles within each time point are technical replicates. 
Table 2 Summary of the additional ChIP-seq profiles analyzed in this study

\begin{tabular}{rcccc}
\hline \multicolumn{5}{c}{ ChIP-seq replicates only } \\
\hline IP factor & E-4-8 $\mathbf{h}$ & AdultMale & AdultFemale & S2 cell \\
\cline { 2 - 5 } & seq & seq & seq & seq \\
\hline CBP & 2 & 2 & 2 & - \\
CTCF & - & - & - & 2 \\
INPUT & 1 & 1 & 1 & 2 \\
\hline
\end{tabular}

Details of the four additional pairs of ChIP-seq/ChIP-seq (biological) replicates analyzed in this study. The two CTCF ChIP-seq profiles were technical replicates, whereas all other ChIP-seq pairs were biological replicates.

and between profiles generated using ChIP-chip and ChIP-seq approaches, and to pinpoint the source of variation between the technologies, which ultimately should provide useful information for experimental design and data analysis.

\section{Results}

\section{Analysis of input DNA profiles}

To understand the technological differences between sequencing-based and microarray-based ChIP data, we first analyzed the profiles of cross-linked and sonicated DNA fragments (input DNA) generated by microarray (INPUT-chip) and high-throughput sequencing (INPUT-seq). Since the input DNA profile should be independent of the antibody used for ChIP, this comparison can give insight into the specific differences between these two profiling technologies. We obtained INPUT-chip data from the background channel of our two-channel microarray data. While this microarray platform uses competitive hybridization, the two channels in our Agilent microarray have been shown to be relatively independent as saturation in either channel is very rare [28]. Out of all the INPUT-chip profiles that we extracted, we only present the analysis of eight representative profiles here (two from each of the four developmental time points) since most of the INPUTchip profiles are very similar (Additional file 2: Figure S1). The eight INPUT-chip profiles were then compared with the nine INPUT-seq profiles collected in this study (Additional file 1: Table S3).

One of the most striking observations is that INPUTchip and INPUT-seq profiles appear to be substantially different, even though the same input DNA material was used for microarray hybridization and sequencing (Figure 1). The relative magnitude and location of the peaks seem to be consistent across the INPUT-chip profiles from multiple experiments. However, the patterns in the nine INPUT-seq profiles appear to be more variable. We can visually identify many regions that have inconsistent signal enrichment across multiple INPUTseq profiles (highlighted in Figure 1a). A clustering analysis was performed to quantify this observation. We found that all eight INPUT-chip profiles clustered closely to one another (Figure $1 \mathrm{~b}$ ). This result shows that the background DNA distribution measured from microarray and high-throughput sequencing is different. All INPUT-chip and seven out of nine INPUT-seq profiles correlated positively with genomic GC content at the genome-wide level (Figure 1b), as well as around the transcription start sites (TSS) and transcription end sites (TES) (Figure 1c). The strength of the correlation with GC is highly consistent among INPUT-chip profiles, but highly variable among the INPUT-seq profiles (Figure $1 \mathrm{~b}-\mathrm{c}$ and Additional file 2: Figure S2). Notably, the INPUT-seq profiles obtained at E-16-20 h (E16) and E-20-24 h (E20) do not correlate with GC content.

We also note that INPUT-seq with higher sequencing depth ( $>4$ million mapped reads) tend to cluster together more tightly than those with lower sequencing depth, suggesting that there may be a relationship between sequencing depth and input DNA variability. To test this hypothesis, we generated 11 additional INPUT-seq profiles by subsampling sequencing reads from the most deeply sequenced input DNA sample (AdultMale; AM) at different sampling proportion (Figure 1d and Additional file 2: Figure S3). As expected, profiles with higher sequencing depth tend to cluster more strongly together, and their correlation with GC content variation is more consistent. However, the GC content correlation only becomes much weaker only at a very low sequencing depth $(<2$ million reads; Figure 1d). This indicates that low sequencing depth is not the only factor affecting INPUT-seq quality. Moreover, some INPUT-seq with relatively low sequencing depth (E0 and AF, $<4$ million reads) can give consistent input DNA profiles. This implies that INPUT-seq variability may also be attributed to other experimental factors. Although further studies are required to dissect the full range of experimental factors affecting variability of input DNA libraries, it could be affected by variations in the sample preparation (e.g., different chromatin preparation and sonication), run-to-run variation of the sequencer, sequencer-to-sequencer variation for the same model, and a host of other variables in experiments. The high variability among INPUT-seq profiles is indeed a critical issue, since large variability contributes to instability of density estimation in a ChIP-seq profile, which will affect downstream data analysis. As will be shown in subsequent sections of this paper, an INPUT-seq with unusually weak correlation with GC content can impact construction of average profiles at important genomic locations. It is thus imperative to sequence the input DNA to sufficient depth and to ascertain that the obtained profile is consistent with those from similar experiments. 


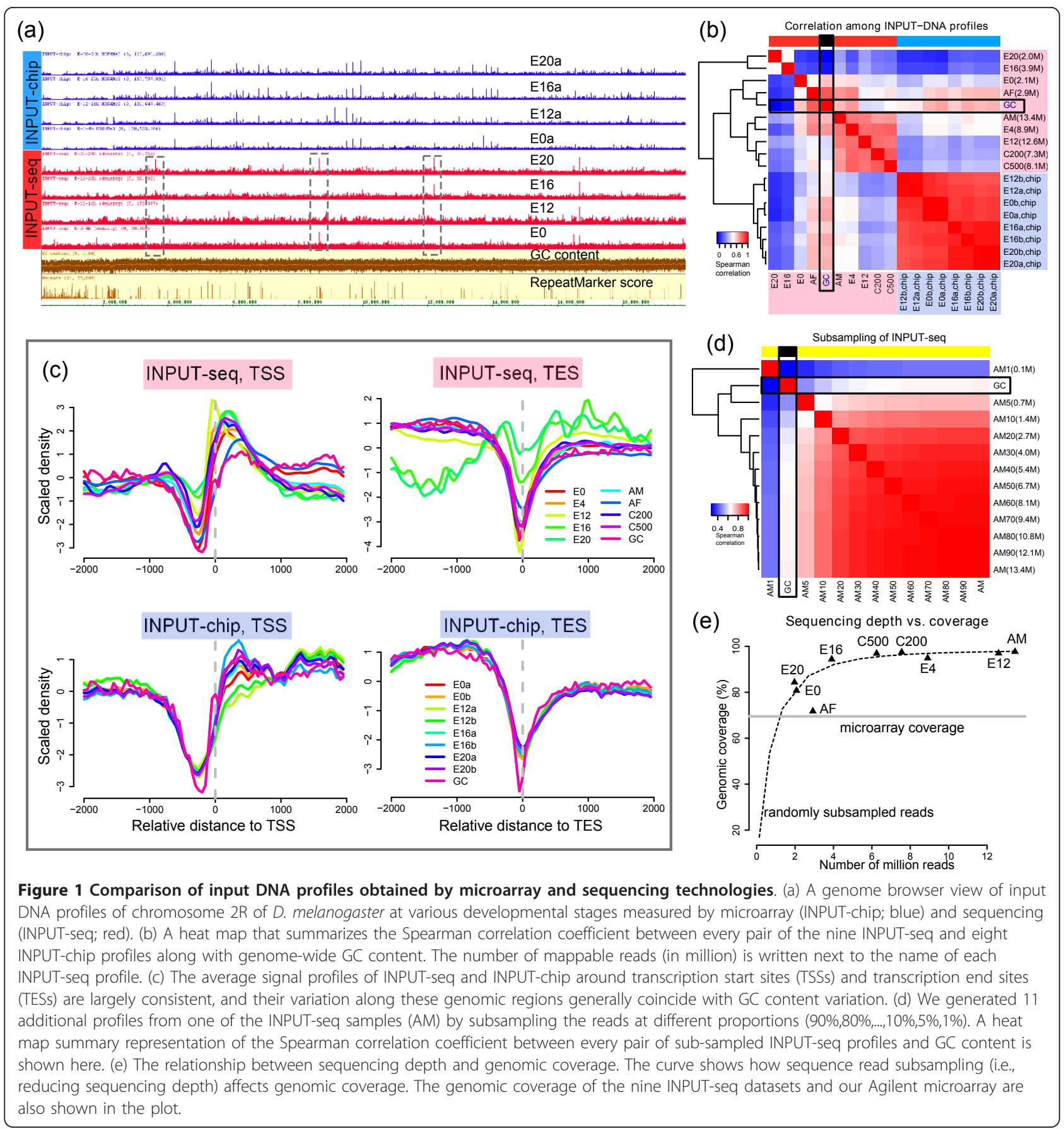

Genomic coverage is another key consideration when choosing between ChIP-chip and ChIP-seq. The genomic coverage of ChIP-chip is limited by the microarray probe design, and the coverage of ChIP-seq is dependent on sequencing depth. The genomic coverage achieved by our Agilent microarray is about 70\%. Using the sub-sampled INPUT-seq data, we show that INPUT-seq generally provides higher genomic coverage at sequencing depth as low as one million reads. This trend constructed from the randomly subsampled data corroborates the observed genomic coverage of the other eight real INPUT-seq datasets (Figure 1e).

\section{Comparison of profile characteristics}

We then compared the characteristics of ChIP-chip and ChIP-seq profiles. To compare the profiles generated by the two technologies, we divided the genome into $1 \mathrm{~kb}$ non-overlapping bins and defined the enrichment level 
at each bin as the average of log ratio of the IP channel over the input channel (see the Methods section for details). We refer to a signal distribution of a ChIP profile as its distribution of enrichment values of all the bins. First, we aimed to characterize the average signalto-noise ratio for profiles generated by the two technologies. We used the (truncated) skewness of the signal density profile after removing signals from the highest and lowest $5 \%$ of the distribution as a measure of signal-to-noise ratio of a profile. Skewness is a measure of asymmetry of a distribution and a positive skewness indicates that the tail on the right side is longer, implying a good signal-to-noise ratio. In almost all cases, a ChIP-seq profile has a higher skewness than its corresponding ChIP-chip profile of the same biological condition (Figure 2 and Additional file 1: Table S4). We note that the difference of skewness is dependent on the IP factor which could be due to different antibody quality and prevalence of histone modification or binding events. The same conclusion can be drawn even if a different bin size was used (Additional file 2: Figure S4). Our results confirmed the general observation that ChIP-seq usually produces a more distinctive signal profile than ChIP-chip.

Next, we characterized the enrichment regions within each ChIP profile. To perform a fair comparison, we would like to use an algorithm that performs peak calling on ChIP-seq and ChIP-chip data using the same criteria. Currently, many commonly used peak calling algorithms are specifically designed for analyzing

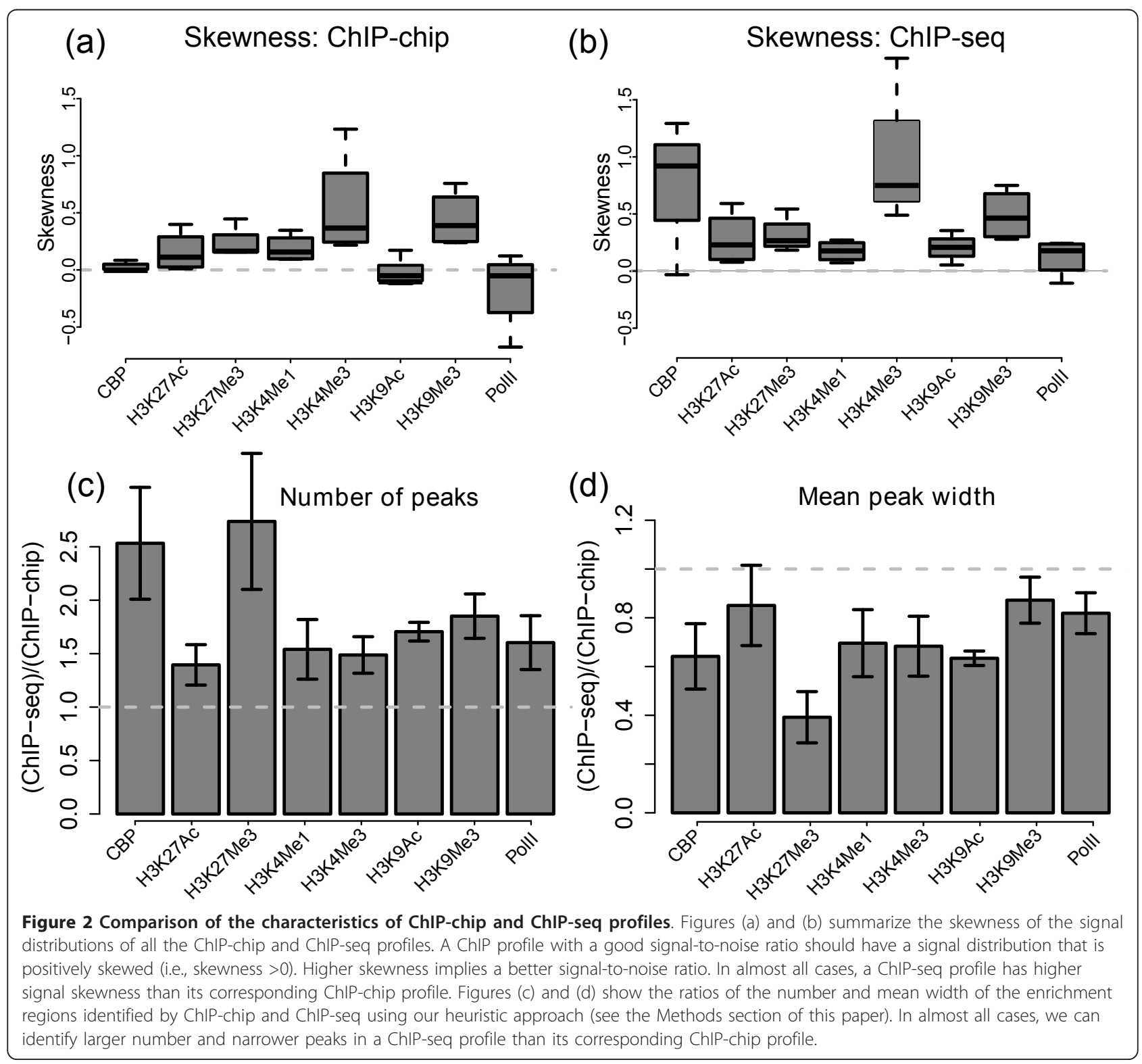


ChIP-chip or ChIP-seq data, but not both. To overcome this limitation, we identified peaks from both ChIP-chip and ChIP-seq profiles using the same genome-scanning heuristic (see the Methods section). Our results indicate that we can almost always discover a larger number of peaks and narrower peaks using data generated from ChIP-seq compared to ChIP-chip when analyzing the same biological sample, and this conclusion is consistent regardless of the stringency of the identification criteria used (Figure 2 and Additional file 2: Figure S5). In practice, we can probably identify an even larger number of narrow peaks in ChIP-seq data if we explicitly make use of strand-specific information within the peak calling procedure (beside only shifting each read towards its 5' end by a constant number of base pair), so the current analysis provides a lower bound on the effectiveness of ChIP-seq compared to ChIP-chip. Taken together, our results demonstrate that ChIP-seq provides higher spatial resolution and signal-to-noise ratio.

\section{Genome-wide signal reproducibility within and between technologies}

Further, we estimated the reproducibility between ChIPchip and/or ChIP-seq profiles at the genome-wide level (1 kb bins). To avoid biases due to differences in genomic coverage and sequence mapping (Figure 1e), we exclude genomic regions that do not contain any microarray probes and regions with unusually high variability across multiple INPUT-seq profiles. The Pearson correlation coefficient, $r$, was used as a measure of correlation, since it is more sensitive than the Spearman correlation coefficient for comparing the tail of two signal distributions, which is particularly important in analyzing ChIP enrichment signal profiles. The correlation between ChIP-chip replicate pairs and between ChIPseq replicate pairs is generally high (median $r=0.85$ and 0.82 , respectively), indicating that both technologies can produce reproducible results. As expected, the cross-platform correlation between replicate pairs of ChIP-chip and ChIP-seq profiles are more modest (median $r=0.41$; Additional file 1: Table S5). Similar conclusions can be reached even if we use different bin sizes for calculating inter-profile correlation (Additional file 2: Figure S6). A representative scatter plot comparing each pair of technologies is shown in Figure $3 \mathrm{~b}-\mathrm{d}$. We also observe a positive correlation between the skewness and inter-profile reproducibility (Additional file 2: Figure S7), suggesting more sensitive antibodies may produce more consistent profiles between the two technologies.

\section{Construction of average signal profile at TSS and TES}

Construction of average ChIP signal profiles around important genomic features such as TSS and TES is a common way to visualize signal enrichment around these features. Therefore, we investigated the reproducibility of average TSS and TES profiles ( $2 \mathrm{~kb}$ up and $2 \mathrm{~kb}$ downstream) for every pair of replicate ChIP profiles (Additional file 2: Figure S8). The average profiles of most replicate pairs are highly consistent. However, there are a few pairs that are significantly different, especially the profiles of $\mathrm{H} 3 \mathrm{~K} 27 \mathrm{Me} 3$ and H3K9Me3 at both stage E-16-20 h and E-20-24 h (Additional file 2: Figures S8c and S8g). Without external validation, it is impossible to determine whether the average signal profiles generated by ChIP-chip or ChIP-seq are more accurate. Nonetheless, two lines of evidence led us to believe that the average signal profiles from ChIP-chip were more likely to be accurate. First, all three ChIP-chip replicates at these time points had very consistent average profiles. Second, the ChIP-seq average signal profiles at these biological conditions resembled the trend of GC content variation at TSS and TES (Figure 1c). The unusually low correlations between GC contents and the INPUT-seq profiles of E-16-20 h and E-20-24 h (Figure $1 \mathrm{~b}$ and Additional file 2: Figure S2b) prompted us to hypothesize that the observed discrepancy was due to a misrepresentation of GC content variation by the respective INPUT-seq profiles. Both H3K27Me3 and H3K9Me3 are repressive marks that are usually depleted at TSSs and TESs so any variation in background subtraction is likely much more pronounced than other histone marks that have strong signal enrichment at these genomic features. To test our hypothesis, we replaced the corresponding INPUT-seq background with the INPUT-seq from the AdultFemale sample, since it has the highest correlation with GC content variation. After the replacement, the average signal profiles generated by ChIP-seq and ChIP-chip at these two developmental stages agree (Figure 4 and Additional file 2: Figure S9). This result is striking since it shows that using different INPUT-seq as negative control of the same ChIP-seq profile can lead to substantially different interpretation of the data.

\section{Effect of using different input profiles in ChIP-seq data normalization}

Having observed the impact of INPUT-seq in constructing average TSS and TES profiles, we asked whether using different INPUT-seq profiles for background normalization significantly affects ChIP-seq peak calling results. We used SPP [29] to call peaks for 10 of our ChIP-seq samples (CBP, H3K9Ac, H3K9Me3, H3K27Ac, H3K27Me3 at E16-20 $\mathrm{h}$ and E20-24 h) where each ChIP profiles was normalized against four different INPUTseq as background (the input from the matching time point, AdultFemale, AdultMale, and E-4-8 h). These INPUT-seq profiles were chosen because they have 


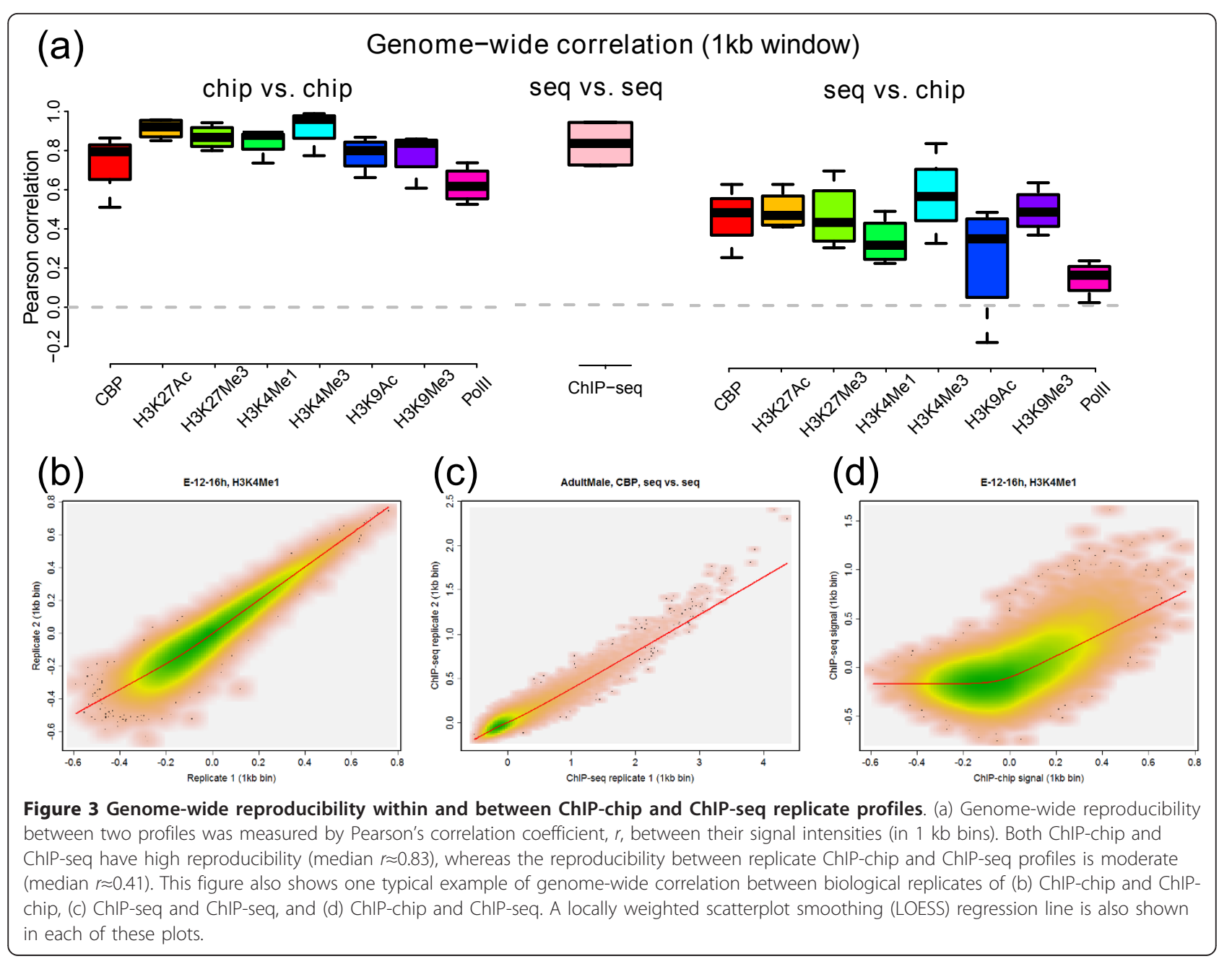

different sequencing depth and correlation with GC content (Figure 1b). A comparison of the number of peaks and median peak width is shown in Figure 5. We observed a large difference in number of peaks being called for any ChIP-seq sample when different INPUTseq was used as background. In the extreme case (E-16$24 \mathrm{~h}, \mathrm{H} 3 \mathrm{~K} 9 \mathrm{Me} 3 \mathrm{ChIP}$ ), the number of peaks can changes from zero to nearly 40,000 at a FDR of 5\% (Figure $5 \mathrm{a}$ ). In general, more statistically significant peaks (FDR $<0.05)$ were detected when normalizing against a deeply sequenced input DNA sample (AdultMale and E4-8 $\mathrm{h}$ in this experiment), although the absolute magnitude of the difference varies among ChIP datasets. The difference in peak number likely indicates a difference in detection power. For each ChIP sample, we calculated the proportion of overlap between each pair of peak sets generated by four different input DNA background (ie, six comparisons per ChIP sample). We found that the mean proportion of overlap with respect to the smaller peak set is about $95 \%$, indicating that the differences in number of detected peak is likely due to different power to call weaker peaks. We observed that the strong peaks (ie, those with low detection FDR) were more likely detected in different peak sets (see Additional file 2: Figure S10 for an example). The median width of the detected peaks is also affected by using different INPUT-seq as background (Figure $5 \mathrm{~b}$ ). This analysis showed that the normalization using different INPUTseq may have a significant, and underappreciated, impact on peak calling.

\section{Assessing variation due to the use of different peak callers}

Another important source of variation in analysis of ChIP-chip and ChIP-seq profiles originates from the use of different analysis algorithms. A large number of publicly available ChIP-chip and ChIP-seq analysis tools have been developed to date [23,30], and all of them utilize different methods for tag shifting, profile normalization, smoothing, peak identification, and calculation of false discovery rate. It is therefore not too surprising to find that different peak callers can generate quite 


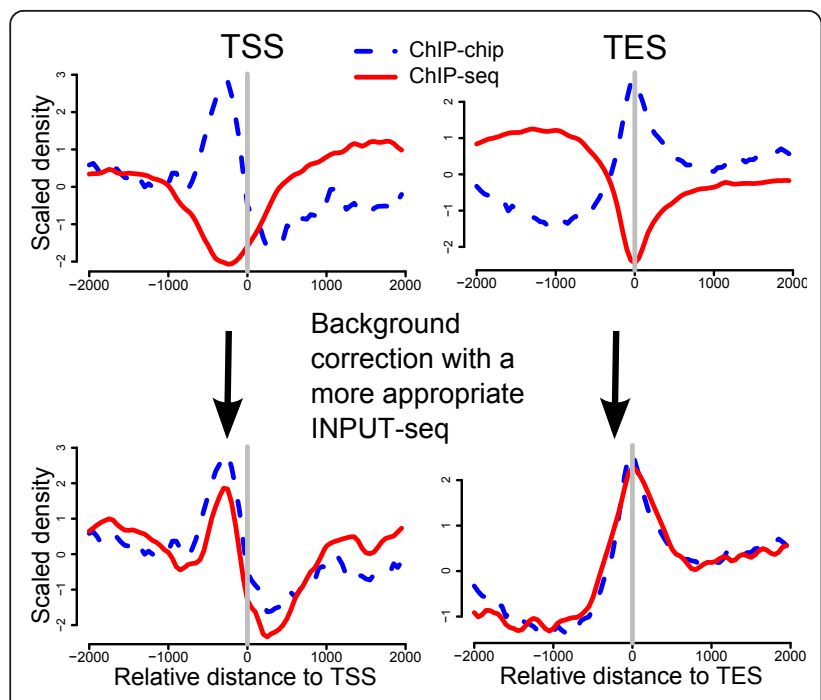

Figure 4 Illustration of how variability in an INPUT-seq profile can affect reconstruction of average signal profile at TSS and TES. The top panel shows the average signal profiles at the TSS and TES for the ChIP-chip and ChIP-seq profiles of H3K27Me3 at E-16-20 h. These ChIP-chip and ChIP-seq profiles differ quite substantially, and the ChIP-seq profiles resemble that of the GC content variation (Figure 1c). We subsequently reprocessed the ChIP-seq sample by using the INPUT-seq at AdultFemale as background for normalization since this profile has a strong correlation with GC content variation, which more likely reflect the actual technologyspecific biases of our sequencing platform. After this procedure, the average signal profiles of ChIP-chip and ChIP-seq look much more alike, indicating that the original INPUT-seq at E-16-20 h does not appropriately capture the technology-specific variation at these sites.

different results in terms of binding site identification, particularly when dealing with peaks with weak signals $[23,31]$. Using our compendium of ChIP-chip and ChIPseq datasets, we could assess how much variation in peak identification can be attributed to the use of different profiling technology and use of different peak callers. In this study, we analyzed our ChIP-chip profiles using two peak callers: MA2C [13] and Splitter [32] and analyzed our ChIP-seq profiles using another two peak callers: MACS [20] and SPP [29] (see Additional file 1: Table S8). These peak callers were chosen because they are widely used, publicly available, and generally show good performance in previous comparative studies $[30,31]$. We calculated the overlap of the top 1,000 peaks of four of the factors (CBP, H3K4Me1, H3K4Me3, and H3K27Me3) across multiple developmental stages. The four IP factors were chosen as they were representative profiles containing broad peaks (CBP and H3K27Me3) and narrow peaks (H3K4Me1 and H3K4Me3). Here, we only present the results of comparing the top 1,000 peaks, since this is a biologically reasonable number of high-confidence enrichment sites in these profiles. The general conclusion of this analysis is robust against a variety of peak calling thresholds
(Additional file 2: Figure S11). Concordance between two peak sets was measured by the average proportion of overlapping peaks. As shown in Figure 6, the comparisons based on profiles of H3K4Me1 and H3K4Me3 yielded expected results, in which the intra-platform concordance is higher than cross-platform concordance (i.e., peak sets generated by two peak callers on the same profile are more concordant than peak sets generated by two peak callers on two profiles). However, the intra-platform concordance can be as low as the interplatform concordance when analyzing the profiles of $\mathrm{H} 3 \mathrm{~K} 27 \mathrm{Me} 3$ and $\mathrm{CBP}$, implying that variation in peak calling algorithms can be as large as the use of different profiling technologies for some IP factors. The observation that current peak calling algorithms produce less concordant results for ChIP profiles with broad domains (CBP and H3K27Me3) than those with sharp peaks (H3K4Me1 and H3K4Me3) may suggest that they are less consistent at identifying broad enrichment regions, which may be an interesting subject for further investigation.

\section{Discussion}

ChIP-seq is an attractive alternative to ChIP-chip due to its many practical advantages. However, to date there is a lack of systematic comparison between ChIP-chip and ChIP-seq based on a large dataset from multiple IP factors. Using a compilation of replicate ChIP-chip and ChIP-seq datasets generated as part of the modENCODE project, we had an unprecedented opportunity to conduct such a systematic comparison. Through comparing the characteristics of the profiles generated by ChIP-chip and ChIP-seq, we showed that ChIP-seq generates profiles with higher signal-to-noise ratios and a larger number of more localized peaks. This is consistent with many previous observations that ChIP-seq generates profiles with higher spatial resolution and dynamic range. Not surprisingly, we found that intertechnology (i.e., ChIP-chip vs. ChIP-seq) reproducibility was lower than intra-technology reproducibility (ChIPchip vs. ChIP-chip or ChIP-seq vs. ChIP-seq). We only had access to four ChIP-seq/ChIP-seq replicate profiles across two IP factors (CBP and CTCF) in this study, so the estimate of intra-platform reproducibility of ChIPseq may be less accurate than that of ChIP-chip. Nonetheless, the overall magnitude of intra-platform reproducibility should still be instructive.

Another important lesson was that variability due to the use of different peak callers can be as substantial as different profiling technologies. Only a small number of representative peak callers were selected in this study, since it was not our primary goal to compare performance of different algorithms. Instead, our goal was to estimate to what extent peak detection variability might 

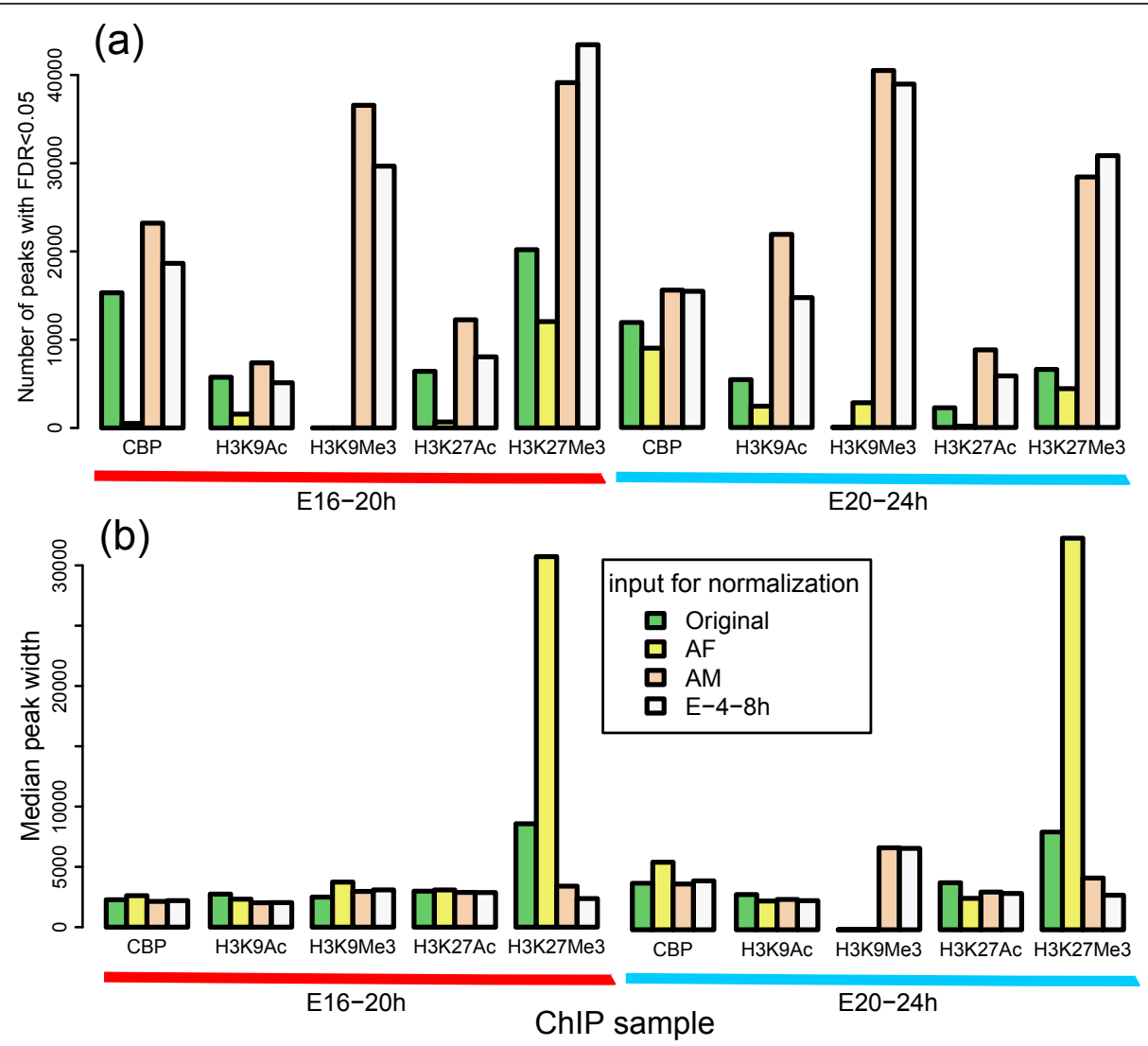

Figure 5 Effect of normalization with different INPUT-seq on ChIP-seq peak calling. We compared the number of peaks (a) and median peak width (b) of $10 \mathrm{ChIP-seq} \mathrm{samples} \mathrm{(CBP,} \mathrm{H3K9Ac,} \mathrm{H3K9Me3,} \mathrm{H3K27Ac,} \mathrm{H3K27Me3} \mathrm{at} \mathrm{E16-20} \mathrm{h} \mathrm{and} \mathrm{E20-24} \mathrm{h)} \mathrm{where} \mathrm{each} \mathrm{of} \mathrm{them} \mathrm{was}$ normalization against four different input DNA samples (the input for from the matching time point, AdultFemale, AdultMale, and E-4-8 h). Peak calling was performed with SPP using the same parameters. Clearly peak detection is significantly affected by using different input DNA library as background control. In general, more peaks are identified as statistically significant (FDR $<0.05)$ when normalized with an INPUT-seq library with higher sequencing depth, although the magnitude of the differences vary across different ChIP datasets.

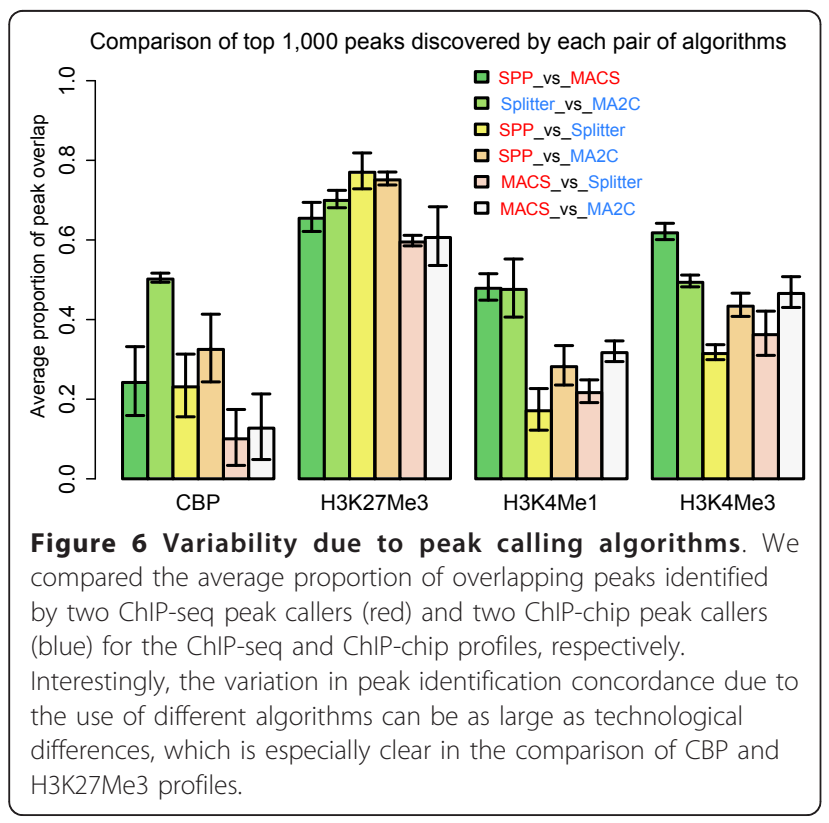

be attributed to the use of different profiling technologies (microarray or sequencing) and use of different peak calling algorithms. The observation that a higher variability in peak detection is associated with broad enrichment domains is interesting, and it requires further assessment using larger numbers of peak callers.

Perhaps one of the most striking findings is that there is high variability among different INPUT-seq compared to INPUT-chip. The differences between INPUT-seq profiles do not seem to correlate with the developmental stages, but rather to sequencing depth (Figure $1 \mathrm{~b}$ and Additional file 2: Figure S3). Nonetheless, sequencing depth alone does not account for all the observed variability. Samples E0, E16, E20 and AF all have low sequencing depth (< 4 million mapped reads), yet AF and E0 have strong correlation with GC content, whereas E20 and E16 have little correlation with GC content (Figure $1 \mathrm{~b}$ and Additional file 2: Figure S3). This suggests that variation in experimental conditions may also contribute to such variability. Our results demonstrate that it is 
important for the input DNA background to capture technology-specific biases, such as GC content variation, as this may have impact on the construction of average signal profiles at important genomic sites. Such average signal profiles have been used as features for building computational models of transcription factor binding or regulatory elements $[33,34]$, so it is important to ensure that the average signal profiles are accurate. In addition, we also show that the quality of input DNA profile used for background normalization when calling peaks for a ChIP-seq dataset is critical (Figure 5). The observation that more peaks were being called when a ChIP-seq dataset was normalized against a more deeply sequenced INPUT-seq suggests that increasing the sequencing depth in the INPUT-seq data may lead to higher statistical power in ChIP-seq peak detection. Currently the importance of input DNA in ChIP-seq analysis is largely underappreciated and most researchers do not even bother to check for the consistency of the input profiles. This work provides quantitative evidence that the success of a ChIP-seq analysis may depend on the quality of input library as much as the quality of ChIP library. Since using an appropriate input DNA profile as background is critical, we believe that obtaining high quality and deeply sequenced input DNA profile is necessary, and that INPUT-seq quality should be assessed more carefully during data analysis.

We recognize there are several limitations in our study. First, we only compared ChIP-chip profiles generated from one commercial platform (Agilent tiling microarray) with ChIP-seq profiles generated from one sequencing platform (Illumina GAII). Although strictly speaking we can only make conclusion about these platforms, we believe that the key lessons learned in this study are instructive for analyzing data generated from other platforms. We note that the Agilent platform uses long oligonucleotides as probes (50-60 mers) and has relatively low noise level compared to other platforms [17,31]; Illumina's GAII sequencing platform is currently the most widely used platform for ChIP-seq, so our analysis should be of interest to many users. Second, unlike previous comparative studies using transcription factor binding data (Additional file 1: Table S1) or "spike in" experiments [31], we do not have external validation of the true enrichment regions, which prohibited us from assessing detection sensitivity and specificity. Nonetheless, we believe that our conclusions, drawn from analyzing many pairs of replicate ChIP-chip/seq profiles, should be reasonably robust.

\section{Conclusions}

Our findings highlight the differences between ChIPchip and ChIP-seq, and show the variability that can arise from both technology and analysis methods. We demonstrate the importance of obtaining high quality and deeply sequenced input DNA libraries for ChIP-seq analysis, which has fundamental implication to experimental design and data analysis.

\section{Methods \\ ChIP-chip and ChIP-seq protocols}

Chromatin immunoprecipitations have been performed as described previously [35]. Briefly, the biological material is homogenized in the presence of $1.8 \%$ formaldehyde. The cross-linked chromatin is sonicated using a Bioruptor (Diagenode) to an average size of $500 \mathrm{bp}$. Precleared chromatin extract is incubated overnight at $4^{\circ} \mathrm{C}$ with the specific antibody and immunoprecipitated with protein-A Sepharose beads. After purification of the DNA and amplification of the libraries by linkermediated PCR, the samples are labeled by incorporating $\mathrm{Cy} 3$ or $\mathrm{Cy} 5$ conjugated dUTPs. Labeled IP and Input samples are hybridized onto the Agilent $1 \mathrm{X} 244 \mathrm{~K}$ arrays using a TecanHS4800Pro at $67^{\circ} \mathrm{C}$ for $24 \mathrm{~h}$. Details related to this custom microarray platform can be found in Additional file 1: Table S9. The non amplified ChIP samples have been used directly for sequencing by Solexa Genome Analyzer following Illumina protocols for libraries generation, cluster generation and sequencing.

\section{Data preprocessing}

For each set of Agilent microarray data, the processed intensity value of the IP and input channels were extracted from the raw data file. For each lane of Illumina sequencing data, the raw sequence reads (36 bp single end) were extracted. Bowtie [36] was used to map both the microarray probe sequences and the Illumina's short reads onto the reference $D$. melanogaster genome assembly ( $\mathrm{dm} 3$, FlyBase built 5.22 ). This procedure ensured that there was no systematic bias due to mapping to different version of the genome assembly. All microarray probe sequences can be uniquely mapped to the genome, and the proportion of mappable reads in our ChIP-seq data is available in Additional file 1: Table S2. For preprocessing of ChIP-seq data, the mapped reads of an IP library and its corresponding input DNA libraries from the same developmental stage were processed by an R package called SPP [29]. In particular, we used SPP to filter out uninformative reads, remove read anomaly and estimate the average fragment length by the cross-correlation profile (Additional file 1: Table S7). We then divided the genome into many 50 bp nonoverlapping bins, and each bin $i$ is associated with a genomic coordinate $x_{i}$ (corresponding to the center of the bin) and an intensity value $y_{i}$ An enrichment value in a bin is the log ratio of the smoothed signal intensity (or smoothed read count for ChIP-seq) of the IP sample 
over the INPUT sample. We used a Gaussian smoother with a bandwidth of 50 for signal smoothing. Specifically, the Gaussian smoother takes the form

$$
y_{i}=\sum_{j} \exp \left[\frac{\left(x^{\prime}{ }_{j}-x_{i}\right)^{2}}{50}\right] y^{\prime}{ }_{j}
$$

where $x_{j}^{\prime}$ and $y_{j}^{\prime}$ are the center of the genomic coordinate and intensity value of probe $j$ in the same chromosome. In practice due to computation constraints, we only consider the 400 closest probes from bin $i$ (200 probes upstream and 200 probes downstream) for smoothing which already give a close approximation to the full Gaussian smoother. Profiles at this 50 bp resolution were used for construction of average profiles of TSS and TES. For other analyses presented in this study, we combined the enrichment value of every 20 adjacent 50 bp bins (by averaging) to obtain a ChIP profile at the $1 \mathrm{~kb}$ resolution. To avoid biases in estimation of genome-wide correlation between two profiles, we first excluded genomic regions that did not contain microarray probes and had high variability in INPUTseq variability. INPUT-chip and INPUT-seq profiles were obtained in a similar manner, except we used the $\log _{2}$ (intensity from input profile) as a measure of enrichment value.

Unless specified otherwise, all data analysis in this study was performed using the $\mathrm{R}$ statistical programming environment [37]. All the signal density profiles were visualized using the Integrated Genome Browser [38].

\section{Construction of average signal profile at TSS and TES}

We used the gene model annotation from FlyBase [39] to define transcription start and end sites. We only included genes with a minimum length of $2 \mathrm{~kb}(7,231$ of 15,186 genes) to exclude short genes in our analysis. The 80 bins ( $50 \mathrm{bp}$ each) surrounding every relevant genomic feature (corresponding to $2 \mathrm{~kb}$ upstream and $2 \mathrm{~kb}$ downstream of the feature) were taken and averaged. The resulting average profiles were scaled such that the mean and the variance of signal in each profile were zero and one, respectively.

\section{Characterization of enrichment signals in ChIP-chip and ChIP-seq profiles}

We calculated skewness of a signal density profile using the following formula:

$$
\operatorname{skewness}\left(x_{1}, x_{2}, \ldots, x_{n}\right)=\frac{\frac{1}{n} \sum_{i=1}^{n}\left(x_{i}-\bar{x}\right)^{3}}{\left[\frac{1}{n} \sum_{i=1}^{n}\left(x_{i}-\bar{x}\right)^{2}\right]^{3 / 2}}
$$

where $x_{i}$ is the enrichment value of bin $i$ of a genome. We specifically removed bins with enrichment value at the lowest or the highest $5 \%$ of the distribution to remove potential outliers.

We devised a simple heuristic method to detect "peaks" that can be identified by ChIP-chip and ChIPseq profiles in a consistent manner. This method consists of two steps: (1) identify candidate enrichment regions using ad hoc criteria, and (2) assign a $p$-value to each candidate enrichment region. For step 1 , we identify all bins with an enrichment value above $\bar{x}+k s$, where $\bar{x}$ and $s$ are, respectively, the sample mean and standard deviation of a signal density per chromosome, and $k$ is an arbitrary parameter. We present the results for $k=0$ in this paper, but we have also performed analysis with $k=-1$ and $k=4$, and the conclusion of the analyses is largely similar (Additional file 2: Figure S5). Adjacent bins are merged to form candidate enrichment regions. For step 2, we assign a statistical significance, $p$, to each enrichment region to be $p=P(l>L)$, where $l$ is the sum of enrichment values of all the bins in the candidate enrichment region, $L \sim N\left(m \bar{x}, m s^{2}\right)$, and $m$ is the number of bins in this enrichment region. To account for multiple comparison, we calculated a false discovery rate (FDR) value for each enrichment region using the method of Benjamini and Hochberg [40], and all the enrichment regions with an FDR less than 0.05 were considered statistically significant. We then characterize each set of enrichment regions from a profile by its number and median width of the enrichment regions.

\section{Additional material}

Additional file 1: Supplemental tables. This file contains supplementary tables.

Additional file 2: Supplemental figures. This file contains supplementary figures.

\section{Acknowledgements}

We thank members of the Park laboratory for constructive comments and suggestions. This work is supported by grants RL1DE019021 and U01HG004258 (PJP), U01HG004264 (KPW), and a SysCODE interdisciplinary postdoctoral training fellowship through Common Fund grant 5RL9EB008539 (JWKH) from the National Institutes of Health.

\section{Author details}

'Department of Medicine, Brigham and Women's Hospital, and Harvard Medical School, Boston, MA, USA. ${ }^{2}$ Center for Biomedical Informatics, Harvard Medical School, Boston, MA, USA. ${ }^{3}$ Program in Bioinformatics, Boston University, Boston, MA, USA. ${ }^{4}$ Institute for Genomics and Systems Biology, University of Chicago, Chicago, IL, USA. ${ }^{5}$ Informatics Program, Children's Hospital, Boston, MA, USA.

Authors' contributions

JWKH designed and performed the study, and wrote the manuscript. EB and PVK performed initial data analysis. NN and KPW generated the ChIP-chip 
and ChIP-seq datasets. PJP conceived, designed, and supervised the study. All authors read and approved the final manuscript.

\section{Competing interests}

The authors declare that they have no competing interests.

Received: 4 January 2011 Accepted: 28 February 2011 Published: 28 February 2011

\section{References}

1. Park PJ: ChIP-seq: advantages and challenges of a maturing technology. Nat Rev Genet 2009, 10:669-680.

2. Lee TI, Rinaldi NJ, Robert F, Odom DT, Bar-Joseph Z, Gerber GK, Hannett NM, Harbison CT, Thompson CM, Simon I, et al: Transcriptional Regulatory Networks in Saccharomyces cerevisiae. Science 2002, 298:799-804

3. Chen $X, X u H$, Yuan $P$, Fang $F$, Huss $M$, Vega VB, Wong E, Orlov YL, Zhang $W$, Jiang J, et al: Integration of external signaling pathways with the core transcriptional network in embryonic stem cells. Cell 2008, 133:1106-1117.

4. Nielsen R, Pedersen TA, Hagenbeek D, Moulos P, Siersbæk R, Megens E, Denissov S, Børgesen M, Francoijs K-J, Mandrup S, et al: Genome-wide profiling of PPARY:RXR and RNA polymerase II occupancy reveals temporal activation of distinct metabolic pathways and changes in RXR dimer composition during adipogenesis. Genes Dev 2008, 22:2953-2967.

5. Barski A, Cuddapah S, Cui K, Roh T, Schones D, Wang Z, Wei G, Chepelev I, Zhao K: High-Resolution Profiling of Histone Methylations in the Human Genome. Cell 2007, 129:823-837.

6. Heintzman ND, Hon GC, Hawkins RD, Kheradpour P, Stark A, Harp LF, Ye Z, Lee LK, Stuart RK, Ching CW, et al: Histone modifications at human enhancers reflect global cell-type-specific gene expression. Nature 2009, 459:108-112

7. Tolstorukov MY, Kharchenko PV, Goldman JA, Kingston RE, Park PJ: Comparative analysis of H2A.Z nucleosome organization in the human and yeast genomes. Genome Res 2009, 19:967-977.

8. Kasowski M, Grubert F, Heffelfinger C, Hariharan M, Asabere A, Waszak SM, Habegger L, Rozowsky J, Shi M, Urban AE, et al: Variation in Transcription Factor Binding Among Humans. Science 2010, 328:232-235.

9. McDaniell R, Lee B-K, Song L, Liu Z, Boyle AP, Erdos MR, Scott LJ, Morken MA, Kucera KS, Battenhouse A, et al: Heritable Individual-Specific and Allele-Specific Chromatin Signatures in Humans. Science 2010, 328:235-239.

10. Ren B, Robert F, Wyrick JJ, Aparicio O, Jennings EG, Simon I, Zeitlinger J, Schreiber J, Hannett N, Kanin E, et al: Genome-Wide Location and Function of DNA Binding Proteins. Science 2000, 290:2306-2309.

11. Johnson WE, Li W, Meyer CA, Gottardo R, Carroll JS, Brown M, Liu XS: Model-based analysis of tiling-arrays for ChIP-chip. Procs Natl Acad SCi USA 2006, 103:12457-12462.

12. Qi Y, Rolfe A, Maclsaac KD, Gerber GK, Pokholok D, Zeitlinger J, Danford T, Dowell RD, Fraenkel E, Jaakkola TS, et al: High-resolution computational models of genome binding events. Nat Biotech 2006, 24:963-970.

13. Song J, Johnson WE, Zhu X, Zhang X, Li W, Manrai A, Liu J, Chen R, Liu XS: Model-based analysis of two-color arrays (MA2C). Genome Biol 2007, 8 R178-R178.

14. Droit A, Cheung C, Gottardo R: rMAT- an R/Bioconductor package for analyzing ChIP-chip experiments. Bioinformatics 2010, 26:678-679.

15. Adli M, Zhu J, Bernstein BE: Genome-wide chromatin maps derived from limited numbers of hematopoietic progenitors. Nat Meth 2010, 7:615-618.

16. Goren A, Ozsolak F, Shoresh N, Ku M, Adli M, Hart C, Gymrek M, Zuk O, Regev A, Milos PM, et al: Chromatin profiling by directly sequencing small quantities of immunoprecipitated DNA. Nat Meth 2010, 7:47-49.

17. Euskirchen GM, Rozowsky JS, Wei C-L, Lee WH, Zhang ZD, Hartman S, Emanuelsson O, Stolc V, Weissman S, Gerstein MB, et al: Mapping of transcription factor binding regions in mammalian cells by ChIP: Comparison of array- and sequencing-based technologies. Genome Res 2007, 17:898-909.

18. Ji H, Jiang H, Ma W, Johnson DS, Myers RM, Wong WH: An integrated software system for analyzing ChIP-chip and ChIP-seq data. Nat Biotech 2008, 26:1293-1300.
19. Rozowsky J, Euskirchen G, Auerbach RK, Zhang ZD, Gibson T, Bjornson R, Carriero N, Snyder M, Gerstein MB: PeakSeq enables systematic scoring of ChIP-seq experiments relative to controls. Nat Biotech 2009, 27:66-75.

20. Zhang Y, Liu T, Meyer C, Eeckhoute J, Johnson D, Bernstein B, Nussbaum C, Myers R, Brown M, Li W, et al: Model-based Analysis of ChIP-Seq (MACS). Genome Biol 2008, 9:R137-R137.

21. Robertson $G$, Hirst M, Bainbridge M, Bilenky M, Zhao $Y$, Zeng $T$, Euskirchen G, Bernier B, Varhol R, Delaney A, et al: Genome-wide profiles of STAT1 DNA association using chromatin immunoprecipitation and massively parallel sequencing. Nat Methods 2007, 4:651-657.

22. Qin ZS, Yu J, Shen J, Maher CA, Hu M, Kalyana-Sundaram S, Yu J, Chinnaiyan AM: HPeak: an HMM-based algorithm for defining readenriched regions in ChIP-seq data. BMC Bioinformatics 2010, 11:369.

23. Laajala T, Raghav S, Tuomela S, Lahesmaa R, Aittokallio T, Elo L: A practical comparison of methods for detecting transcription factor binding sites in ChIP-seq experiments. BMC Genomics 2009, 10:618-618.

24. Teytelman L, Özaydin B, Zill O, Lefrançois P, Snyder M, Rine J, Eisen MB: Impact of Chromatin Structures on DNA Processing for Genomic Analyses. PLOS ONE 2009, 4:e6700-e6700.

25. Vega VB, Cheung E, Palanisamy N, Sung W-K: Inherent Signals in Sequencing-Based Chromatin-ImmunoPrecipitation Control Libraries. PLOS ONE 2009, 4:e5241-e5241.

26. Auerbach RK, Euskirchen G, Rozowsky J, Lamarre-Vincent N, Mogtaderi Z, Lefrancois P, Struhl K, Gerstein M, Snyder M: Mapping accessible chromatin regions using Sono-Seq. Proc Natl Acad Sci USA 2009, 106:14926-14931

27. Celniker SE, Dillon LAL, Gerstein MB, Gunsalus KC, Henikoff S, Karpen GH, Kellis M, Lai EC, Lieb JD, MacAlpine DM, et al: Unlocking the secrets of the genome. Nature 2009, 459:927-930

28. Zahurak M, Parmigiani G, Yu W, Scharpf R, Berman D, Schaeffer E, Shabbeer S, Cope L: Pre-processing Agilent microarray data. BMC Bioinformatics 2007, 8:142-142.

29. Kharchenko PV, Tolstorukov MY, Park PJ: Design and analysis of ChIP-seq experiments for DNA-binding proteins. Nat Biotech 2008, 26:1351-1359.

30. Wilbanks EG, Facciotti MT: Evaluation of Algorithm Performance in ChIPSeq Peak Detection. PLOS ONE 2010, 5:e11471-e11471.

31. Johnson DS, Li W, Gordon DB, Bhattacharjee A, Curry B, Ghosh J, Brizuela L, Carroll JS, Brown M, Flicek $P$, et al: Systematic evaluation of variability in ChIP-chip experiments using predefined DNA targets. Genome Res 2008, 18:393-403.

32. Splitter. [http://zlab.bu.edu/splitter]

33. Won K-J, Ren B, Wang W: Genome-wide prediction of transcription factor binding sites using an integrated model. Genome Biol 2010, 11:R7-R7.

34. Firpi HA, Ucar D, Tan K: Discover Regulatory DNA Elements Using Chromatin Signatures and Artificial Neural Network. Bioinformatics 2010, 26:1579-1586.

35. Negre N, Lavrov S, Hennetin J, Bellis M, Cavalli G: Mapping the distribution of chromatin proteins by ChIP on chip. Methods Enzymol 2006, 410:316-341

36. Langmead B, Trapnell C, Pop M, Salzberg S: Ultrafast and memory-efficient alignment of short DNA sequences to the human genome. Genome Biol 2009, 10:R25-R25.

37. R: A language and environment for statistical computing. [http://www.Rproject.org].

38. Nicol JW, Helt GA, Blanchard SG, Raja A, Loraine AE: The Integrated Genome Browser: free software for distribution and exploration of genome-scale datasets. Bioinformatics 2009, 25:2730-2731.

39. Drysdale RA, Crosby MA, The FlyBase C: FlyBase: genes and gene models. Nucl Acids Res 2005, 33, D390-395-D390-395.

40. Benjamini $Y$, Hochberg Y: Controlling the False Discovery Rate: A Practical and Powerful Approach to Multiple Testing. J R Stat Soc Ser B 1995, 57:289-300.

doi:10.1186/1471-2164-12-134

Cite this article as: Ho et al:: ChIP-chip versus ChIP-seq: Lessons for experimental design and data analysis. BMC Genomics 2011 12:134. 